\title{
Proliferation of metanephric mesenchymal cells is inhibited by miR-743a-mediated WT1 suppression in vitro
}

\author{
MINGMING XUE ${ }^{1,2}$, YURU ZHOU ${ }^{3}$, XIAOYU LIU $^{1}$, DONGSHENG NI ${ }^{1}$, YANXIA HU ${ }^{1}$, \\ YAOSHUI LONG ${ }^{1}$, PAN JU ${ }^{1}$ and QIN ZHOU ${ }^{1}$ \\ ${ }^{1}$ Division of Molecular Nephrology and The Creative Training Center for Undergraduates, \\ M.O.E. Key Laboratory of Laboratory Medical Diagnostics, College of Laboratory Medicine, \\ Chongqing Medical University, Chongqing 400016; ${ }^{2}$ Laboratory of Molecular Pathology, College of Basic Medicine, \\ Inner Mongolia Medical University, Inner Mongolia 010000; ${ }^{3}$ The Seventh Class of 2012 Year Entry, \\ Third Clinical College, Chongqing Medical University, Chongqing 400016, P.R. China
}

Received September 10, 2015; Accepted September 8, 2016

DOI: $10.3892 / \mathrm{mmr} .2016 .5762$

\begin{abstract}
To seek out the potential microRNAs (miRNAs) that target Wilms' tumor suppressor 1 (WT1), a transcription factor required for progenitor proliferation as well as normal development of the kidney, and to clarify the effects of the miRNAs on WT1, the 3'-untranslated region (3'-UTR) of WT1 was initially analyzed and miR-743a, a seldom-reported miRNA, was identified. In the present paper, luciferase reporter assays were performed to confirm that miR-743a is able to directly target the 3'-UTR of WT1. Subsequently, reverse transcription-quantitative polymerase chain reaction, combined with western blotting analyses, were performed, and the results revealed a significant inhibition of WT1 at the mRNA and the protein levels. Furthermore, a 5-ethynyl-2'-deoxyuridine (EdU) cell proliferation assay, coupled with a WT1 rescue strategy, demonstrated that miR-743a inhibited the proliferation of metanephric mesenchymal (MM) cells, in part by targeting WT1. In conclusion, by targeting WT1, miR-743a suppresses the proliferation of MM cells in vitro, and probably possesses vital functions in kidney development and kidney-associated diseases.
\end{abstract}

\section{Introduction}

The development of the mammalian kidney occurs via a series of precisely controlled processes. Derived from a region of the mesoderm, the kidney is gradually formed from the

Correspondence to: Professor Qin Zhou, Division of Molecular Nephrology and The Creative Training Center for Undergraduates, M.O.E. Key Laboratory of Laboratory Medical Diagnostics, College of Laboratory Medicine, Chongqing Medical University, 1 Yixueyuan Road, Yuzhong, Chongqing 400016, P.R. China E-mail: zhouqin@cqmu.edu.cn; pkdzhou@126.com

Key words: miR-743a, Wilms' tumor suppressor 1, proliferation, metanephric mesenchymal, kidney metanephric mesenchymal (MM) cells and the ureteric bud (UB). Furthermore, under the reciprocal induction of complex molecular regulatory processes, the kidney with the entirety of its functions comes into being (1-3). In the early development of the kidney, Wilms' tumor suppressor 1 (WT1), a zinc-finger transcription factor, is expressed in the MM cells, cap mesenchyme (CM), and further during the course of nephron development. WT1 exerts an important role in maintaining the self-renewal of MM cells, and the well-balanced development of the kidney (4-7). Loss of WT1 results in embryonic lethality and hypoplasia of the gonads, together with bilateral renal agenesis, which is characterized by an incomplete formation of the UB and concurrent apoptosis of the MM cells $(7,8)$. Therefore, these findings suggested that WT1 is intrinsically required for the functioning of the MM cells, and is indispensable for the completion of the kidney developmental program (9), and consequently, further research on WT1 regulation is urgently required.

In addition to the regulation of coding genes, there also exists non-coding RNA regulation. MicroRNAs (miRNAs) are a class of endogenous non-coding RNAs that regulate gene expression either through translational repression or via mRNA degradation by binding to the 3'-untranslated region (3'-UTR) of the targeted mRNAs $(10,11)$. MiRNAs exert important roles in development, proliferation, apoptosis and other biological processes (12). Based on previous studies, miRNA-dependent gene regulation serves an important role in kidney development and disease (13). For example, miR-181 was demonstrated to downregulate Six homeobox 2 (Six2) protein and inhibit the proliferation of MM cells $(14,15)$; miR-135 mediated kidney podocyte injury through $\mathrm{Wnt} / \beta$-catenin signaling (16); miR-17 promoted cell proliferation through polycystin-2 (PKD2) (17); and depletion of miR-150 suppressed acute kidney injury induced by myocardial infarction (18). Of the miRNAs, miR-743a has rarely been reported, and therefore its function has yet to be fully elucidated. The present study therefore aimed to explore the biological function of miR-743a in kidney development.

In the current study, miR-743a has been demonstrated to directly target the 3'-UTR of WT1, and to downregulate 
the expression of WT1 at the mRNA and the protein level. Furthermore, miR-743a is revealed to inhibit the proliferation of MM cells. These results have disclosed the functional effects of miR-743a in kidney development, and opened up novel means for investigating potential cures for kidney diseases in which WT1 exerts a participatory role.

\section{Materials and methods}

Plasmid construction. The plasmids, pcDNA3.1-luciferaseWT1-3'-UTR-WT, pcDNA3.1-luciferase-WT1-3'-UTR-MUT, pdsAAV-CB-EGFP-miR-743a and pRL-SV40, were used in dual-luciferase assays, as described below. The 3'-UTR of WT1, together with miR-743a, was cloned from C57BL/6 mouse genomic DNA using polymerase chain reaction (PCR). C57BL $/ 6$ mouse tail was digested with proteinase $\mathrm{K}$ at $55^{\circ} \mathrm{C}$ with vibration overnight, and centrifuged at $13,800 \times \mathrm{g}$ for $5 \mathrm{~min}$ at room temperature and then the supernatant was collected to obtain genomic DNA. The mouse was obtained from the animal centre of Chongqing Medical University (Chongqing, China), and the use of the mouse was approved by the Animal Care and Use Committee of Chongqing Medical University. The vector, pcDNA3.1(+)-WT1-CDS, was obtained by PCR from the complementary DNA (cDNA) of mK3 cells (an immortalized cell line of undifferentiated MM cells), and was recombined at the restriction sites of BamHI and EcoRI to construct pcDNA3.1 (+)-WT1-CDS. Details of the primers used in the present study are provided in Table I.

Cell culture. 293T cells, as well as mK3 cells, obtained from the American Type Culture Collection (Manassas, VA, USA) were cultured in Dulbecco's modified Eagle's medium [DMEM (Gibco; Thermo Fisher Scientific, Inc., Waltham, MA, USA)], with 10\% FBS (Gemini 900-108) and penicillin/streptomycin (Gibco; Thermo Fisher Scientific, Inc.) at $37^{\circ} \mathrm{C}$ with $5 \% \mathrm{CO}_{2}$.

Transfection. The plasmids, pcDNA3.1-luciferase-WT1 -3'-UTR-WT, pcDNA3.1-luciferase-WT1-3'-UTR-MUT, pdsAAV-CB-EGFP-miR-743a and pRL-SV40 were transfected in 293T cells via the calcium phosphate transfection method. When 293T cells reached 50\% confluence, $2.5 \mu \mathrm{l}$ $2.5 \mathrm{M} \mathrm{CaCl}_{2}$ was mixed with $500 \mathrm{ng}$ pcDNA3.1-luciferas e-WT1-3'-UTR-WT or $500 \mathrm{ng}$ pcDNA3.1-luciferase-WT1 -3'-UTR-MUT and $500 \mathrm{ng}$ pdsAAV-CB-EGFP or $500 \mathrm{ng}$ pdsAAV-CB-EGFP-miR-743a together with $10 \mathrm{ng}$ pRL-SV40, then isovolumetric $2 \mathrm{X}$ Hank's balanced salt solution (16.3 g NaCl, $0.74 \mathrm{~g} \mathrm{KCl}, 0.214 \mathrm{~g} \mathrm{Na}_{2} \mathrm{HPO}_{4}, 2.4 \mathrm{~g}$ glucose, $10 \mathrm{~g}$ HEPES, adjusted to $\mathrm{pH} 7.05$ ) was added to the mixture above nad inubated for $2 \mathrm{~min}$ at room temperature. The mixture was subsequently added to the cells in 24-well plates in serum-free medium. The plasmids, pcDNA3.1 (+)-WT1-CDS and miR-743a mimic or control mimic (50 nM) (Guangzhou RiboBio Co., Ltd., Guangzhou, China) were transfected into mK3 cells using Lipofectamine ${ }^{\circledR}-2000$ (Invitrogen; Thermo Fisher Scientific, Inc.). Following transfection, the medium was replaced with fresh complete medium after $6 \mathrm{~h}$ and after $48 \mathrm{~h}$, luciferase activity was measured using the Dual-Luciferase Reporter assay system (Promega Corporation, Madison, WI, USA).

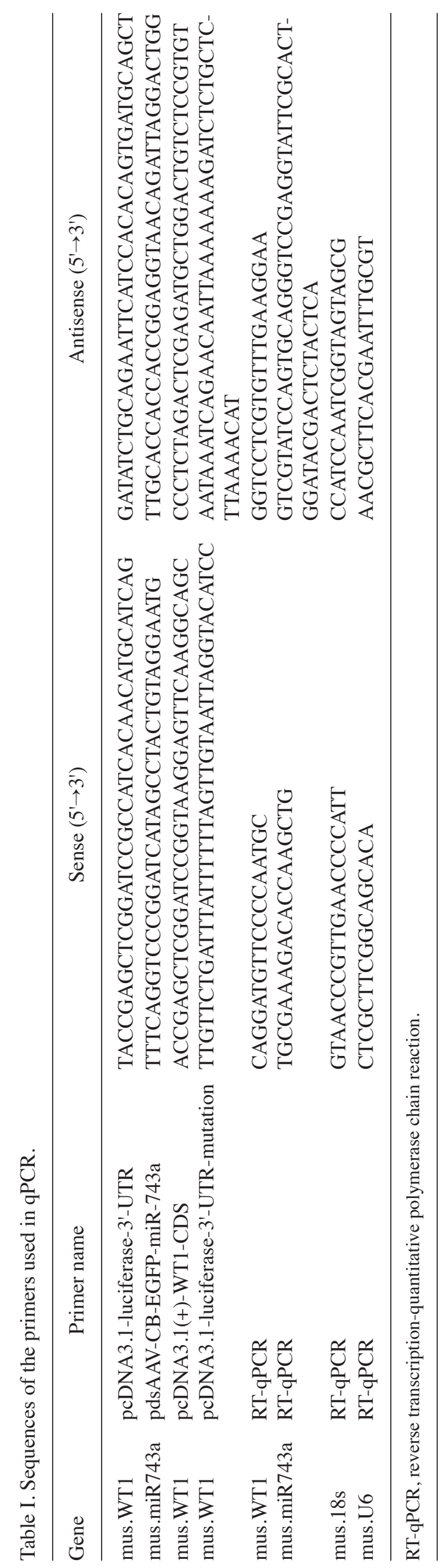


Dual-luciferase assays. Luciferase assays were performed following the method described in a previous study (14). The plasmids, pdsAAV-CB-EGFP-miR-743a and pRL-SV40, were co-transfected with pcDNA3.1-luciferase or pcDNA3.1-luciferase-WT1-3'-UTR or pcDNA3.1-luci ferase-WT1-3'-UTR-mutant using the calcium phosphate method. After $48 \mathrm{~h}$, luciferase activity was measured using the Dual-Luciferase ${ }^{\circledR}$ Reporter Assay system, following the manufacturer's protocol.

$R N A$ extraction and reverse transcription-quantitative $P C R$ $(R T-q P C R)$. Total mK3 RNA was collected with TRIzol ${ }^{\circledR}$ reagent (Invitrogen; Thermo Fisher Scientific, Inc.). The cDNA was reverse-transcribed using the RevertAid First-Strand cDNA Synthesis kit (cat. no. K1622; Thermo Fisher Scientific, Inc.), and RT-qPCR experiments were performed using the SYBR Premix Ex Taq ${ }^{\mathrm{TM}}$ II kit (Takara Biotechnology Co., Ltd., Dalian, China) and cycled at $95^{\circ} \mathrm{C}$ for $10 \mathrm{~min}$ followed by 39 cycles of $95^{\circ} \mathrm{C}$ for $15 \mathrm{sec}$ and $60^{\circ} \mathrm{C}$ for $1 \mathrm{~min}$. The expression level of WT1 mRNA was normalized against the internal control (18S), whereas the expression of miR-743a was normalized against U6. The primers are provided in Table I. The expression levels were normalized using the $\Delta \Delta \mathrm{Cq}$ method (19).

Western blot analysis. MiR-743a mimic $(50 \mathrm{nM})$ or control mimic $(50 \mathrm{nM})$ was transfected into $\mathrm{mK} 3$ cells, and, after $48 \mathrm{~h}$, the cells were rinsed with $1 \mathrm{X}$ phosphate-buffered saline three times and subsequently lysed with radioimmunoprecipitation assay (RIPA) lysis buffer (Beyotime Institute of Biotechnology, Beijing, China). The cells were incubated on ice for $15 \mathrm{~min}$ and then ruptured by ultrasonication. The lysates were centrifuged at $2-8^{\circ} \mathrm{C}, 13,800 \times \mathrm{g}$ for $20 \mathrm{~min}$, and subsequently boiled with $5 \mathrm{X}$ SDS loading buffer at $95^{\circ} \mathrm{C}$ for $10 \mathrm{~min}$. The proteins were separated using $12 \%$ SDS-PAGE. The proteins were transferred to polyvinylidene fluoride membrane and then blocked with $5 \%$ non-fat powdered milk in TBS-Tween (1.21 g Tris, $5.84 \mathrm{~g} \mathrm{NaCl}$, adjusted to $\mathrm{pH} 7.4$, in $1 \mathrm{~L} \mathrm{ddH}_{2} \mathrm{O}$, with $0.1 \%$ Tween-20) at room temperature for $2 \mathrm{~h}$. The membranes were incubated with rabbit anti-WT1 (cat. no. sc-192; 1:1,000 dilution, Santa Cruz Biotechnology, Inc., Santa Cruz, CA, USA) and mouse anti- $\beta$-actin (cat. no. HC201-02; 1:2,000 dilution, Beijing Transgen Biotech Co., Ltd., Beijing, China) at $4^{\circ} \mathrm{C}$ overnight then washed with TBS-Tween three times for $10 \mathrm{~min}$. The blots were incubated with peroxidase-conjugated Affinipure goat anti-rabbit immunoglobulin $\mathrm{G}[(\mathrm{IgG}), \mathrm{H}+\mathrm{L}$, cat. no. 10285-1-AP; 1:5,000 dilution, ProteinTech Group, Inc., Chicago, IL, USA) for $2 \mathrm{~h}$ at room temperature. Images were detected using the ChemiDoc ${ }^{\mathrm{TM}} \mathrm{XRS}+$ system of Bio-Rad (Bio-Rad Laboratories, Inc., Hercules, CA, USA).

Cell proliferation assay. At $36 \mathrm{~h}$ after transfection, the extent of proliferation of the $\mathrm{mK} 3$ cells was determined on a glass slide using a Cell-Light EdU Apollo 567 in vitro kit (Guangzhou RiboBio Co., Ltd., Guangzhou, China), according to the manufacturer's protocol. The visual fields were observed under a fluorescence inverted microscope (Nikon, Japan). The ratio of red cells (EdU-staining-positive cells) to the blue cells (total cells labeled by DAPI) was identified as the proliferation rate.
Bioinformatic analysis. The evolutionary conservation of WT1 3'UTR was obtained from The University of California Santa Cruz Genome Browser. The potential miRNAs that target WT1 were predicted by the cited prediction programs using miRWalk (www.microwalk.org), microRNA.org and TargetScan (/www.targetscan.org).

Statistical analysis. All the experiments were performed in triplicate, and the results and analyses are presented as the means \pm standard deviation and as a paired $t$-test. GraphPad Prism5 software (GraphPad, San Diego, CA, USA) was used to analyze the data and to construct the bar charts. $\mathrm{P}<0.05$ was considered to indicate a statistically significant difference.

\section{Results}

Bioinformatics analysis of WT1 3'-UTR. To analyze the conservation of the 3'-UTR of WT1, a BLAST search was performed on the sequences across a variety of species through the online bioinformatics site [The University of California Santa Cruz Genome Browser]. As shown in Fig. 1A, the 3'-UTR of WT1 is reasonably well conserved across different species, including rat, human, rabbit and dog. Subsequently, the potential miRNAs were screened using three online bioinformatics tools (miRWalk, microRNA.org and TargetScan). The preliminary results revealed that miR-743a is likely to bind the 3'-UTR of WT1, and the binding sites are also conserved to a certain extent (Fig. 1B). To the best of our knowledge, up to this point, the role of miR-743a in, and its possible association with, kidney development has rarely been reported. These data suggested that the 3'-UTR of WT1 is conserved among vertebrates to a certain extent, and that miR-743a is potentially able to target it.

miR-743a directly targets the 3'-UTR of WT1. To investigate whether miR-743a is able to directly target the 3 '-UTR of WT1, the plasmids, pcDNA3.1-luciferase-WT1-3'-UTR and pdsAAV-CB-EGFP-miR-743a, were constructed (Fig. 2A). The plasmids, pcDNA3.1-luciferase-WT1-3'-UTR and pdsAAV-CB-EGFP-miR-743a or pdsAAV-CB-EGFP were co-transfected into $293 \mathrm{~T}$ cells. At the same time, the plasmid pRL-SV40 was included as an internal reference. Compared with the control transfected with pdsAAV-CB-EGFP, overexpression of miR-743a led to a downregulation in relative luciferase activity (Fig. 2B). When the binding sites of miR-743a on the 3'-UTR of WT1 were scrambled (Fig. 2A), no significant difference in the activity of luciferase was identified following the transfection with miR-743a (Fig. 2B). These data indicated that miR-743a is able to directly target the 3'-UTR of WT1, leading to a decrease in the reporter gene activity.

miR-743a suppresses the expression of WT1. To further explore the effects of miR-743a on WT1 in mK3 cells (an immortalized cell line of undifferentiated MM cells), control mimic or miR-743a mimic $(50 \mathrm{nM})$ was transfected into mK3 cells. After $48 \mathrm{~h}$, the cells were collected in order to determine the mRNA and protein expression levels of WT1. The cells transfected with miR-743a mimic revealed a marked increase in miR-743a expression at the mRNA level (Fig. 3A). However, the expression of WT1 was clearly decreased when 
A
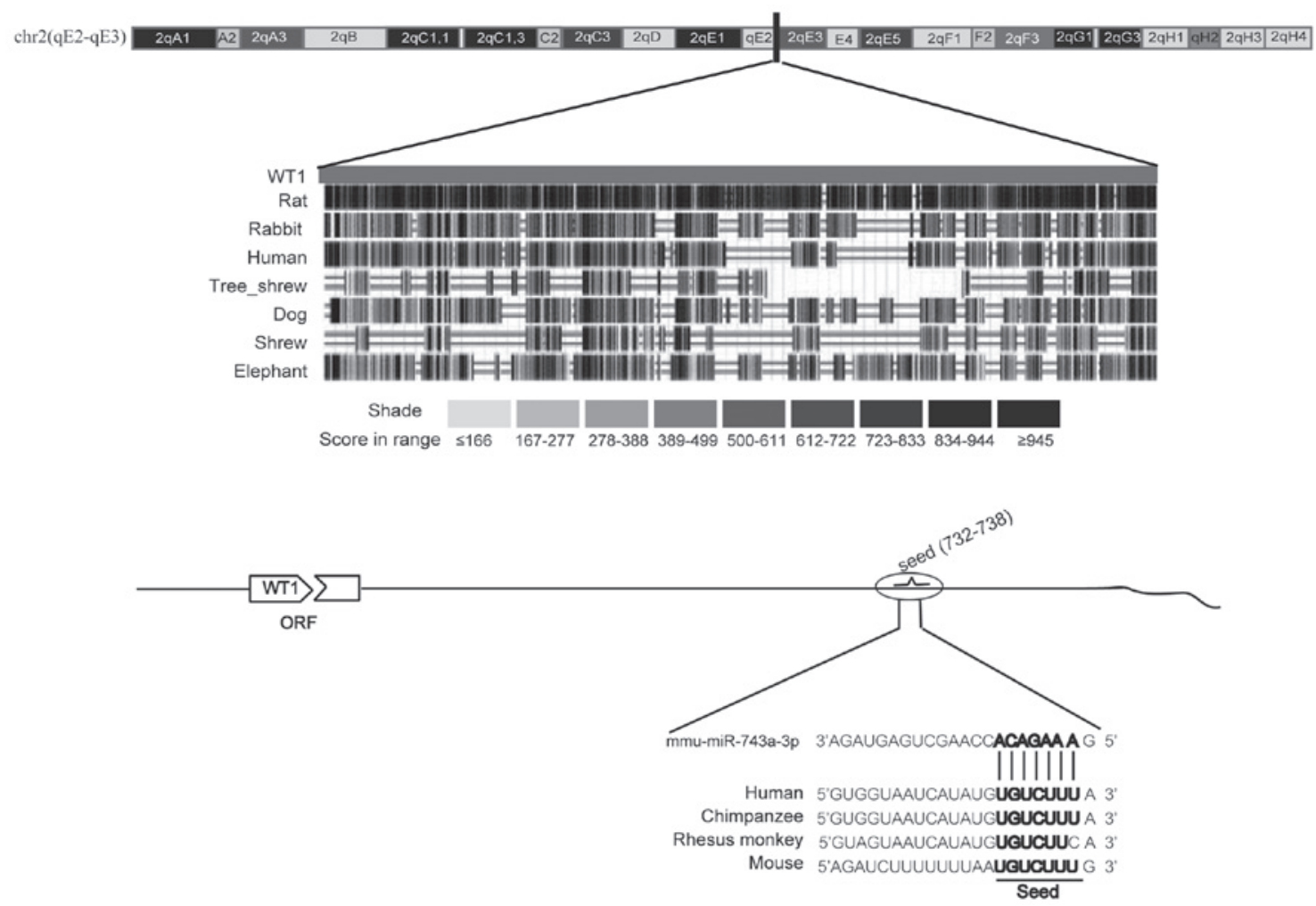

Figure 1. Bioinformatics analysis of the 3'-UTR of WT1. (A) The figure demonstrates that the 3'-UTR of WT1 is located on chromosome 2 (assessed using the University of California Santa Cruz Genome Browser), and the extent of conservation of the sequence is illustrated by the differential shading (from pale gray through to black, which signifies the higher level of conservation), as scored by phastCons. (B) A diagram showing the 3'-UTR of WT1 and the predicted binding sites of mir-743a, prepared using the bioinformatics website, TargetScan release 6.2 database. 3'-UTR, 3'-untranslated region. WT1, Wilms' tumor suppressor $1, \mathrm{ORF}$, open reading frame.

transfected with miR-743a compared with the control group, as demonstrated using RT-qPCR (Fig. 3B). Furthermore, the protein level of WT1 also revealed a marked decrease, as revealed by western blot analysis (Fig. 3C and D). Taken together, these data suggested that miR-743a is able to downregulate the expression of WT1 at the mRNA and the protein level.

Proliferation of MM cells may be attenuated by miR-743a, and rescued in part by WT1. To verify whether miR-743a is able to affect the proliferation of $\mathrm{mK} 3$ cells via WT1, miR-743a mimics or negative control mimics $(50 \mathrm{nM})$ were transfected into $\mathrm{mK} 3$ cells. After $36 \mathrm{~h}$, the proliferation was measured using an EdU kit. As shown in Fig. 4A-C, the proliferation rate was markedly decreased in cells transfected with miR-743a together with pCNDA3.1(+). Furthermore, as shown in Fig. 4A-C, the proliferation rate was rescued when the cells overexpressing miR-743a were transfected with WT1. These data suggested that miR-743a is able to inhibit the proliferation of MM cells, and that this may be partially rescued by WT1.

\section{Discussion}

Three important findings are associated with the present study: i) miR-743a is able to directly target WT1; ii) miR-743a is able to decrease the expression of WT1 at the mRNA and the protein level; and iii) miR-743a inhibits the proliferation of MM cells via WT1. To the best of our knowledge, these

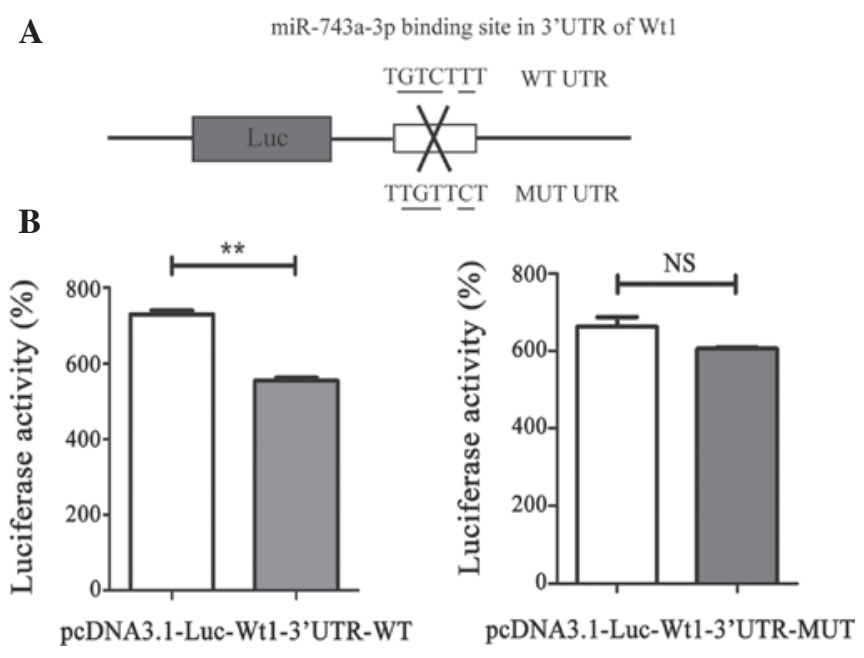

Figure 2. miR-743a directly targets the 3'-UTR of WT1. (A) The reporter vector contains the 3'-UTR of WT1. Two fragments of WT1 3'-UTR, including wild-type as well as the mutant type, are formed. (B) Transfection of the plasmids, pcDNA3.1-luciferase-3'-UTR and pdsAAV-CB-EGFP-miR-743a, together with pRL-SV40 reporter, into $293 \mathrm{~T}$ cells is shown (the control group: pcDNA3.1-luciferase-3'-UTR, pdsAAV-CB-EGFP and pRL-SV40). After $48 \mathrm{~h}$, the cells were lysed and the fluorescence was detected in order to assess the activity, normalized against Renilla activity. $\mathrm{P}<0.01$ compared with the control. 3'-UTR, 3'-untranslated region; WT1, Wilms' tumor suppressor 1; Luc, luciferase; WT, wild type; NS, not significant.

mechanistic details have been established for the first time in the search to identify the important functions of miR-743a in kidney development. 
A
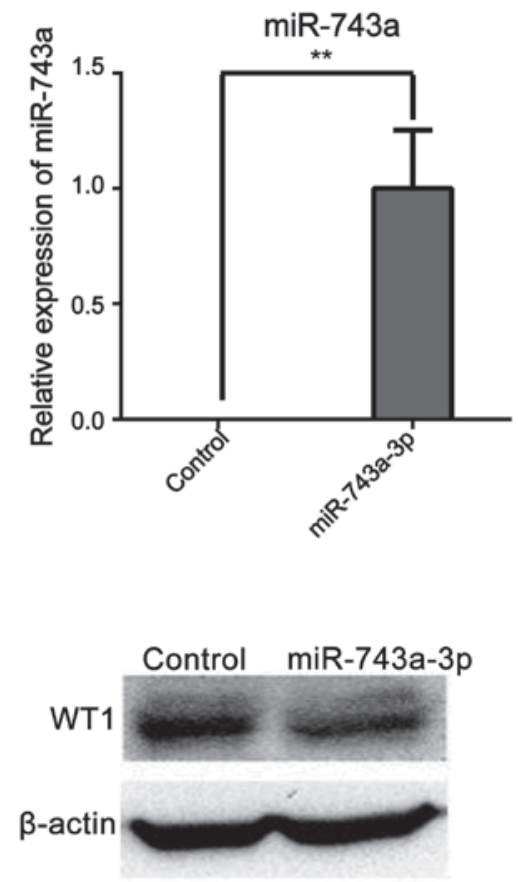

B

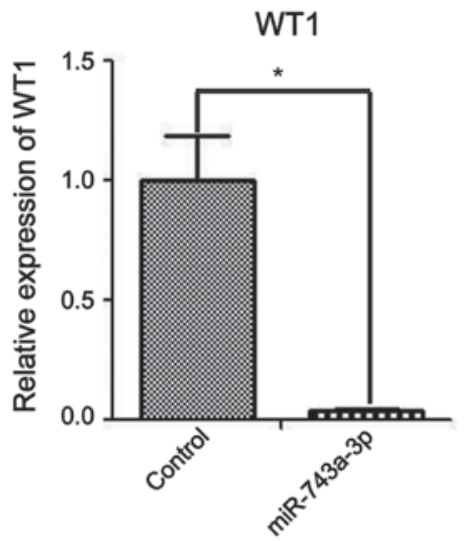

D

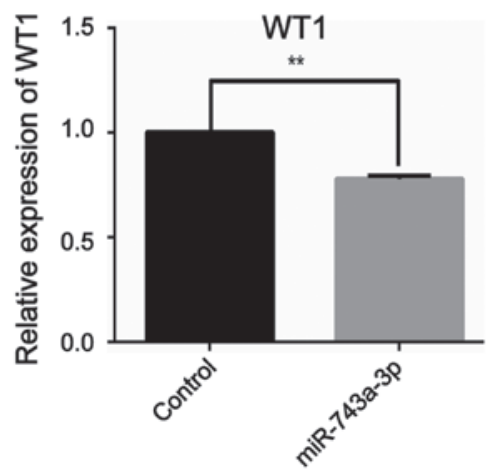

Figure 3. miR-743a downregulates the expression of WT1. (A) Detection of miR-743a mRNA expression by RT-qPCR following transfection with miR-743a mimic in mK3 cells. (B) Overexpression of miR-743a reduced the mRNA level of endogenous WT1. (C) Detection of WT1 protein expression by Western blotting. Overexpression of miR-743a reduced the protein level of endogenous WT1. (D) Densitometric analysis of the Western blotting data shown. "P<0.05; ${ }^{* *} \mathrm{P}<0.01$, compared with the control. WT1, Wilms' tumor suppressor protein 1; RT-qPCR, reverse transcription-quantitative polymerase chain reaction.

A
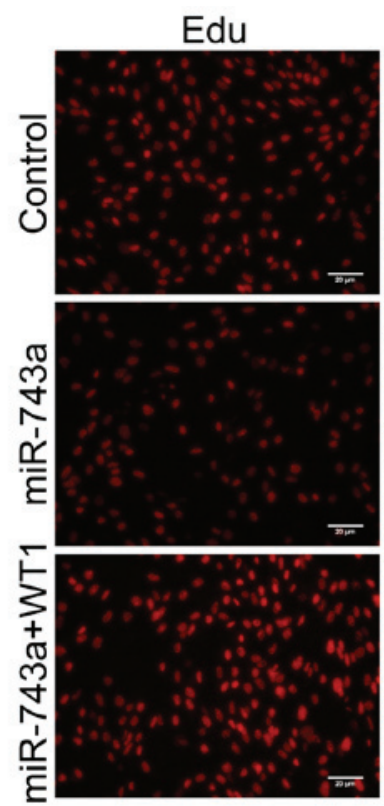

DAPI
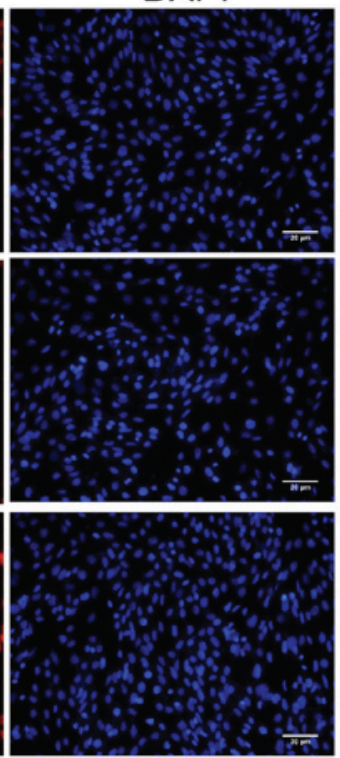

Merge

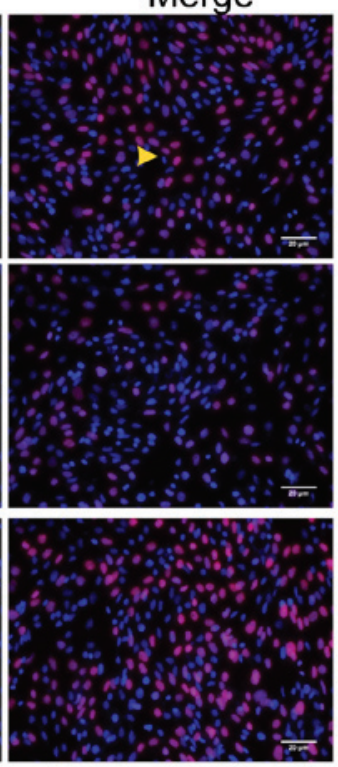

B

\section{Edu}

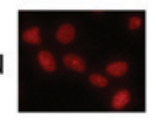

DAPI

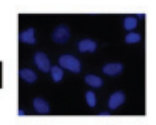

Merge

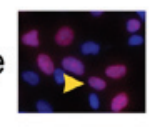

C

Edu

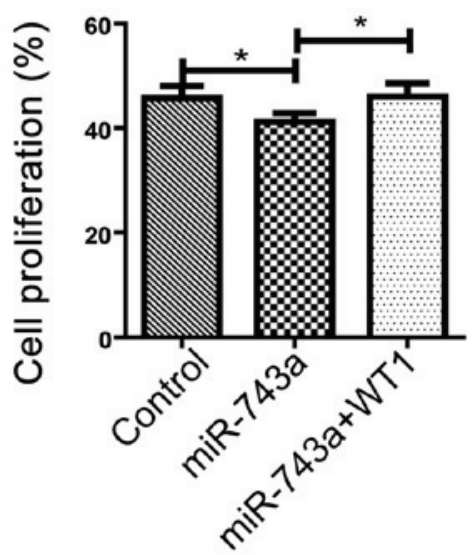

Figure 4. miR-743a inhibits the proliferation of MM cells through WT1. (A) The EdU fields were assessed using inverted fluorescence microscopy (magnification, $\mathrm{x} 200$ ), and the $\mathrm{mK} 3$ cells were stained in red with EdU, and in blue with DAPI. (B) The red coloration represents cell proliferation, whereas the blue staining is representative of the cell number. (C) The relative counting statistics of the EdU proliferation assay are shown. "P $<0.05$. Data are reported as mean \pm standard deviation of the mean for three independent experiments. WT1, Wilms' tumor suppressor 1; EdU, 5-ethynyl-2'-deoxyuridine; MM cells, metanephric mesenchymal cells. 
WT1 was originally described in Wilm's tumor, a condition that is characterized by undifferentiated blastema, stromal cells, and even structures such as epithelium in developing kidneys (20). In addition to Wilm's tumors, mutation of WT1 can result in other severe kidney diseases. WT1 fulfills important roles in maintaining the proliferation of MM cells and in the balance of kidney development (21). In addition, WT1 controls a series of progenitor-specific genes in the kidney, including Sall1, Pax2, Wnt8b, and so forth $(4,21)$. A proportion of reported kidney failures are caused by WT1 mutations, which consequently creates public health problems worldwide (22). Abnormal WT1 protein results in the dysfunction of either renal progenitors or podocytes, which ultimately lead to renal agenesis (22). Therefore, an investigation of the upstream regulation of WT1 becomes a priority.

Other than code gene regulation in kidney development, regulation of non-coding RNA is also functionally important in the kidney. To the best of our knowledge, there is a scarcity of reports on miR-743a, and particularly regarding its function in the kidney In the present study, it has been demonstrated that miR-743a may directly target the 3'-UTR of WT1. For the first time, the important function of miR-743a has been identified in the kidney, and the foundations have been laid for further study of the role of miR-743a in kidney development and disease.

In conclusion, in the current study it has been identified that miR-743a targets WT1 in kidney MM cells. Furthermore, the mechanism underpinning miR-743a inhibition of the proliferation of MM cells through WT1 has been partly elucidated. Future studies will focus on the possible role of miR-743a in kidney diseases, with a view to elucidating new leads in curing kidney diseases caused by WT1.

\section{Acknowledgements}

We would like to thank all the members of the Division of Molecular Nephrology and the Creative Training Center for Undergraduates, The M.O.E. Key Laboratory of Laboratory Medical Diagnostics, The College of Laboratory Medicine, Chongqing Medical University, for their assistance. This work was funded by the National Basic Research Program of China (grant no. 2011CB944002 to Professor Qin Zhou) and The National Natural Science Foundation of China (grant nos. 31271563 and 81572076 to Professor Qin Zhou).

\section{References}

1. Dressler GR: The cellular basis of kidney development. Annu Rev Cell Dev Biol 22: 509-529, 2006.

2. Little MH and McMahon AP: Mammalian kidney development: Principles, progress, and projections. Cold Spring Harb Perspect Biol 4: pii: a008300, 2012.
3. Lindström NO, Lawrence ML, Burn SF, Johansson JA, Bakker ER, Ridgway RA, Chang CH, Karolak MJ, Oxburgh L, Headon DJ, et al: Integrated $\beta$-catenin, BMP, PTEN, and Notch signalling patterns the nephron. Elife 3: e04000, 2015.

4. Motamedi FJ, Badro DA, Clarkson M, Lecca MR, Bradford ST, Buske FA, Saar K, Hübner N, Brändli AW and Schedl A: WT1 controls antagonistic FGF and BMP-pSMAD pathways in early renal progenitors. Nat Commun 5: 4444, 2014.

5. Armstrong JF, Pritchard-Jones K, Bickmore WA, Hastie ND and Bard JB: The expression of the Wilms' tumour gene, WT1, in the developing mammalian embryo. Mech Dev 40: 85-97, 1993.

6. Pritchard-Jones K, Fleming S, Davidson D, Bickmore W, Porteous D, Gosden C, Bard J, Buckler A, Pelletier J, Housman D, et al: The candidate Wilms' tumour gene is involved in genitourinary development. Nature 346: 194-197, 1990.

7. Berry RL, Ozdemir DD, Aronow B, Lindström NO, Dudnakova T, Thornburn A,Perry P, Baldock R, Armit C, Joshi A, et al: Deducing the stage of origin of Wilms' tumours from a developmental series of Wt1-mutant mice. Dis Model Mech 8: 903-917, 2015.

8. Kirschner KM, Braun JF, Jacobi CL, Rudigier LJ, Persson AB and Scholz $\mathrm{H}$ : Amine oxidase copper-containing 1 (AOC1) is a downstream target gene of the Wilms tumor protein, WT1, during kidney development. J Biol Chem 289: 24452-24462, 2014.

9. Donovan MJ, Natoli TA, Sainio K, Amstutz A, Jaenisch R, Sariola H and Kreidberg JA: Initial differentiation of the metanephric mesenchyme is independent of WT1 and the ureteric bud. Dev Genet 24: 252-262, 1999.

10. Cannell IG, Kong YW and Bushell M: How do microRNAs regulate gene expression? Biochem Soc Trans 36: 1224-1231, 2008.

11. Wang Z: miRNA in the regulation of ion channel/transporter expression. Compr Physiol 3: 599-653, 2013.

12. Alvarez-Garcia I and Miska EA: MicroRNA functions in animal development and human disease. Development 132: 4653-4662, 2005.

13. Ho J and Kreidberg JA: The long and short of microRNAs in the kidney. J Am Soc Nephrol 23: 400-404, 2012.

14. lyu Z, Mao Z, Wang H, Fang Y, Chen T, Wan Q, Wang M, Wang N, Xiao J, Wei H, et al: MiR-181b targets Six 2 and inhibits the proliferation of metanephric mesenchymal cells in vitro. Biochem Biophys Res Commun 440: 495-501, 2013.

15. Lv X, Mao Z, Lyu Z, Zhang P, Zhan A, Wang J, Yang H, Li M, Wang H, Wan Q, et al: miR181c promotes apoptosis and suppresses proliferation of metanephric mesenchyme cells by targeting Six2 in vitro. Cell Biochem Funct 32: 571-579, 2014.

16. Yang X, Wang X, Nie F, Liu T, Yu X, Wang H, Li Q, Peng R, Mao Z, Zhou Q and Li G: miR-135 family members mediate podocyte injury through the activation of $\mathrm{Wnt} / \beta$-catenin signaling. Int J Mol Med 36: 669-677, 2015.

17. Sun H, Li QW, Lv XY, Ai JZ, Yang QT, Duan JJ, Bian GH, Xiao Y, Wang YD, Zhang Z, et al: MicroRNA-17 post-transcriptionally regulates polycystic kidney disease- 2 gene and promotes cell proliferation. Mol Biol Rep 37: 2951-2958, 2010.

18. Ranganathan P, Jayakumar C, Tang Y, Park KM, Teoh JP, Su H, Li J, Kim IM and Ramesh G: MicroRNA-150 deletion in mice protects kidney from myocardial infarction-induced acute kidney injury. Am J Physiol Renal Physiol 309: F551-F558, 2015.

19. Livak KJ and Schmittgen TD: Analysis of relative gene expression data using real-time quantitative PCR and the 2(-Delta Delta C(T)) Method. Methods 25: 402-408, 2001.

20. Hastie ND: The genetics of Wilms' tumor - a case of disrupted development. Annu Rev Genet 28: 523-558, 1994.

21. Dong L, Pietsch S and Englert C: Towards an understanding of kidney diseases associated with WT1 mutations. Kidney Int 88: 684-690, 2015.

22. Eckardt KU, Coresh J, Devuyst O, Johnson RJ, Köttgen A, Levey AS and Levin A: Evolving importance of kidney disease: From subspecialty to global health burden. Lancet 382: 158-169, 2013. 